\title{
miR-451 suppresses the malignant characteristics of colorectal cancer via targeting SAMD4B
}

\author{
CHUNRONG WU ${ }^{*}$, XIAOHU LIU ${ }^{2 *}$, BO LI $^{3}$, GUIYIN SUN $^{1}$, CHUNFANG PENG $^{1}$ and DEBING XIANG ${ }^{1}$ \\ Departments of ${ }^{1}$ Oncology, ${ }^{2}$ Gastrointestinal Surgery and ${ }^{3}$ Cardiology, \\ Jiangjin Central Hospital of Chongqing, Chongqing 402260, P.R. China
}

Received July 14, 2020; Accepted March 23, 2021

DOI: $10.3892 / \mathrm{mmr} .2021 .12196$

\begin{abstract}
Cancer metastasis and recurrence are major causes of poor survival in patients with colorectal cancer (CRC). Therefore, the biological behavior of microRNA (miR)-451 in CRC deserves further investigation. Reverse transcription-quantitative PCR was applied to measure the relative expression of miR-451 in blood serum specimens from patients with CRC and CRC cells. In vitro, HCT116 cells were transfected with miR-451 mimics, a miR-451 inhibitor, or SAMD4B plasmids. Proliferation, migration and apoptosis were measured using CCK-8, Transwell assays and flow cytometry, respectively. Luciferase reporter assay was used to identify targets of miR-451 and western blotting performed to explore the internal mechanisms of miR-451 regulation. In vivo, the effect of miR-451 and SAMD4B plasmids on tumor growth was analyzed using a nude mouse xenograft model. Results indicated that serum miR-451 expression was lower in patients with CRC compared with healthy controls. Patients with elevated expression of miR-451 had longer survival times compared with those with low expression. Overexpression of miR-451 inhibited proliferation and migration, promoted apoptosis and enhanced the sensitivity of CRC cells to chemotherapy. SAMD4B was identified as a direct target of miR-451 using miRNA target prediction programs and dual luciferase reporter assay validated the binding site of miR-451 in the 3-'UTR region of SAMD4B. Further studies confirmed that miR-451 inhibited CRC progression via targeting SAMD4B.
\end{abstract}

Correspondence to: Dr Bo Li, Department of Cardiology, Jiangjin Central Hospital of Chongqing, 725 Jiangzhou Avenue, Dingshan Street, Jiangjin, Chongqing 402260, P.R. China

E-mail: libobo1255@163.com

Professor Debing Xiang, Department of Oncology, Jiangjin Central Hospital of Chongqing, 725 Jiangzhou Avenue, Dingshan Street, Jiangjin, Chongqing 402260, P.R. China

E-mail: xdb86@hotmail.com

*Contributed equally

Key words: miR-451, colorectal cancer, proliferation, migration, apoptosis, SAMD4B
Results indicated that miR-451 is essential for blocking tumor growth via targeting SAMD4B in vivo and in vitro. The miR-451/SAMD4B axis may serve as a novel therapeutic target in patients with CRC.

\section{Introduction}

Colorectal cancer (CRC) is one of the commonest types of cancer and a leading cause of cancer mortality worldwide (1). It is well established that the pathogenesis of CRC involves genetic and epigenetic changes and an imbalance in oncogenes and tumor suppressor genes (2). With the improvement of early screening strategies and CRC treatment methods, such as surgery, chemotherapy, or pre-operative neoadjuvant chemoradiotherapy plus surgery, the survival rate of patients with CRC has improved. However, the prognosis for advanced $\mathrm{CRC}$ remains poor. Chemoresistance is the main reason for treatment failure and disease progression (3). The survival of patients is significantly reduced because of consequent tumor recurrence and distant metastasis (4). Therefore, further identification of key molecules, which not only regulate the development and progression of CRC but may also be used as early detection biomarkers or novel therapeutic targets, would be of great benefit to patients with CRC.

MicroRNAs (miRNAs/miRs) belong to a group of non-coding single-stranded small RNA molecules that contain $\sim 22$ nucleotides and are highly conserved (5). miRNAs perform modulatory functions in a series of biological processes $(6,7)$. Increasing evidence indicates that miRNA may be promising biomarkers for disease diagnosis and prognosis $(8,9)$ and dysregulation of miRNA may contribute to the development and progression of cancer (10-12). As miRNAs exist stably in the blood and blood samples are easy to obtain, the detection of circulating miRNAs in human bodily fluids such as serum has become more broadly studied in research (13). As serum miRNAs expression profiles in tumor patients differ significantly from that of healthy individuals $(14,15)$, serum miRNAs may be considered promising biomarkers for tumor detection $(16,17)$.

As a tumor suppressor gene, miR-451 inhibits the survival, proliferation and invasion of cancer cells and serves a significant role in tumor development (18-20). Furthermore, studies focused on multiple solid tumor types have shown that miR-451 may be a meaningful biomarker for cancer diagnosis, 
treatment and drug resistance (21-23). Several studies have focused on the decreased expression of miR-451, which is closely associated with CRC progression $(24,25)$. However, the underlying molecular mechanisms remain understudied.

The present study was designed to detect the expression of miR-451 in patients with CRC and CRC cells and to determine the role of miR-451 in CRC cells. The targets of miR-451 that induce the related signaling pathway changes were explored. The findings demonstrated that miR-451 is essential to blocking tumor growth via targeting SAMD4B.

\section{Materials and methods}

Cell culture and human blood serum specimens. CRC cells (HT29, SW480 and HCT116) and normal colorectal mucosal cell lines (FHC and HCoEpiC) were purchased from the American Type Culture Collection in March 2018 and grown in DMEM media (Sigma-Aldrich; Merck KGaA) with $10 \%$ fetal bovine serum (HyClone; Cytiva). The HT-29 cell line was authenticated by STR profiling. Cells were cultured in a humidified incubator at $37^{\circ} \mathrm{C}$ with $5 \% \mathrm{CO}_{2}$. Oxaliplatin was purchased from Jiangsu Hengrui Pharmaceutical Co., Ltd.; 5-Fluorouracil (5-FU) was purchased from Shanxi Pude Pharmaceutical Co., Ltd.

Serum was collected and pre-processed in the Departments of Gastrointestinal Surgery and Oncology of Jiangjin Central Hospital of Chongqing prior to surgical treatment. The process of extracting serum was performed as described by Allen et al (14). A total of 50 diagnostic patient specimens were used in this study (Table I). Serum from 50 healthy subjects served as the control group (Table II). The Ethics Committee of the Jiangjin Central Hospital of Chongqing approved the research protocol (approval no. 20190611-28) and informed written consent was received from each patient.

Transfection experiments. For cell function research, negative control (NC), synthetic miR-451 mimics and an miR-451 inhibitor were purchased from Guangzhou RiboBio Co., Ltd. The SAMD4B overexpression plasmid and the scramble plasmid were purchased from Shanghai GeneChem Co., Ltd. The process of cell transfection was performed as described by Fan and Zhao (26). Transfection was performed using Lipofectamine ${ }^{\circledR} 3000$ Reagent (Thermo Fisher Scientific, Inc.) in accordance with the manufacturer's protocols. In brief, HCT 116 cells were cultured in 6 -well plates at $37^{\circ} \mathrm{C}$ until they reached $60-70 \%$ confluence. Then $\sim 4 \mu \mathrm{g}$ transfectant [negative control (NC), synthetic miR-451 mimics, miR-451 inhibitor, SAMD4B overexpression plasmid, or scramble plasmid] were added. Cells were collected for subsequent experiments after $24 \mathrm{~h}$ co-culture at $37^{\circ} \mathrm{C}$. The oligonucleotide sequences were as follows: hsa-miR-451 mimics, 5'-AAACCGUUACCAUUA CUGAGUU-3'; mimics NC, 5'-TTCTCCGAACGTGTCACG T-3'; miR-451 inhibitor, 5'-AACUCAGUAAUGGUAACG GUUU-3'; and inhibitor NC, 5'-CAGUACUUUUGUGUA GUACAA-3' (all Guangzhou RiboBio Co., Ltd.). The plasmid vector was pcDNA 3.1, and the over expression sequences of SAMD 4B was 5'-ACTGGAGGACCGCAACGCAC-3' (Shanghai GeneChem Co., Ltd.).

In vivo experiments. A total of six female athymic (nu/nu) mice per group were purchased from the Laboratory
Animal Center of Chongqing Medical University [certificate. SCXK(YU)2018-0003]. A total of 24 mice (age, 6 weeks) were maintained in polycarbonate cages (temperature, $21-23^{\circ} \mathrm{C}$; humidity, 40-60\%; 5 animals per cage) on a 12-h light/dark cycle. All of the animal procedures were approved by the Ethics Committee of the Chongqing Medical University (approval no. 2019-256). Mice were utilized to generate a xenograft tumor model and each group included six mice. They were injected subcutaneously with $1 \times 10^{6}$ HCT116 cells that were stably transfected with i) negative control (NC), ii) lentiviral vectors with miR-451, iii) lentiviral vectors with SAMD4B, or iv) lentiviral vectors with miR-451 plus SAMD4B. The lentiviral vector system included the pCDH-CMV-EF1-co pGFP-T2A-SAMD4B-puro and the pcDNA3.1 plasmids, which were purchased from Shanghai GeneChem Co., Ltd. The process of transfection was performed as described by Zhu et al (27). In brief, HCT 116 cells were cultured in 6-well plates to $60-70 \%$ confluence. A total of $100 \mu \mathrm{l}$ opti-MEM (Gibco; Thermo Fisher Scientific, Inc.) was used to dissolve $2.5 \mu \mathrm{g}$ plasmid; the same volume was also added to $5 \mu \mathrm{l}$ Lipofectamine 3000 (Thermo Fisher Scientific, Inc.), then incubated at room temperature for $5 \mathrm{~min}$. Lastly, the two solutions were mixed and incubated at room temperature for $15 \mathrm{~min}$, then added to the cells. After $72 \mathrm{~h}$ transfection at $37^{\circ} \mathrm{C}$, neomycin $(450 \mu \mathrm{g} / \mathrm{ml})$ was used for selection. Following transfection, $1 \times 10^{6}$ HCT116 cells in $100 \mu 1$ serum-free medium were injected subcutaneously into each mouse (right back). The subcutaneous tumor size of each mouse was measured weekly with an electronic caliper. Mice were sacrificed when the tumor size reached $1,500 \mathrm{~mm}^{3}$, the largest diameter of a tumor was $19 \mathrm{~mm}$ and the mice were sacrificed using cervical dislocation and the xenograft tumor tissues were excised and weighed to determine the tumor weights. Tumor volume was measured as following: $1 / 2 \mathrm{x}$ a (the largest diameter) $\mathrm{x}^{2}$ (the perpendicular diameter).

Cell viability analysis. Cells were transfected with the indicated plasmid or drugs (Oxaliplatin was purchased from Jiangsu Hengrui Pharmaceutical Co., Ltd., and 5-FU). Oxaliplatin was used at a concentration gradient of $2,4,8,12,24$ and $48 \mu \mathrm{M}$. 5-FU was used at a concentration gradient of 1, 2, 4, 8, 16 and $32 \mu \mathrm{g} / \mathrm{ml}$ at 8,24, 48 and $72 \mathrm{~h}$ after miR-451mimics and inhibitors transfection and evaluation of cell proliferation via cell viability was determined by a Cell Counting Kit- 8 (CCK-8; MedChemExpress). The cell viability was measured using optical density (OD) at $450 \mathrm{~nm}$.

$R N A$ extraction and reverse transcription-quantitative $(R T-q)$ $P C R$. Blood samples were collected in BD Vacutainer Serum Separation Tubes, incubated for $1 \mathrm{~h}$ at room temperature and centrifuged at $1,300 \mathrm{x} \mathrm{g}$ for $10 \mathrm{~min}$ at $4^{\circ} \mathrm{C}$. The serum supernatant was transferred to new tubes, centrifuged at $12,000 \mathrm{x} \mathrm{g}$ for $10 \mathrm{~min}$ at $4^{\circ} \mathrm{C}$ to remove any residual cells and debris and stored at $-80^{\circ} \mathrm{C}$. The serum was collected from fasting patients and controls and, for patients, the serum was collected before the first cycle of chemotherapy. RNA was extracted from cells or human blood serum specimens using TRIzol ${ }^{\circledR}$ reagent (Thermo Fisher Scientific, Inc.) and RNA concentration was measured on a NanoDrop spectrophotometer (Thermo Fisher Scientific, Inc.). RT-qPCR was conducted according to the 
Table I. Clinical features of patients enrolled in the present study.

\begin{tabular}{ll} 
Characteristic & $\mathrm{n}=$ \\
\hline Age & \\
$\geq 60$ & 33 \\
$<60$ & 17 \\
Sex & 31 \\
Male & 19 \\
Female & \\
Pathology & 28 \\
High-moderately differentiated adenocarcinoma & 22 \\
Poorly differentiated adenocarcinoma & \\
Stage & 15 \\
I-II & 35 \\
III-IV & \\
Type of therapy & 39 \\
Adjuvant chemotherapy & 11 \\
Neoadjuvant chemotherapy & \\
\hline
\end{tabular}

manufacturer's protocol. miR-451, U6, SAMD4B and GAPDH expression were measured by RT-qPCR using the primer set from Guangzhou RiboBio Co., Ltd. The primer sequences for these genes are given in Table III. miRNA was reverse transcribed using the polyA tailing method (All-in-One ${ }^{\mathrm{TM}}$ miRNA RT-qPCR detection kit), while mRNA was reverse transcribed using the All-in-One ${ }^{\mathrm{TM}}$ First-Strand cDNA Synthesis kit (both from GeneCopoeia, Inc.) and then the DNAse digestion on RNA extracts. The following temperature protocol was used: Incubation at $37^{\circ} \mathrm{C}$ for $60 \mathrm{~min}$ and $72^{\circ} \mathrm{C}$ for $5 \mathrm{~min}$; the samples were stored at $4^{\circ} \mathrm{C}$. The following thermocycling conditions were used: Initial denaturation at $95^{\circ} \mathrm{C}$ for $10 \mathrm{~min}$, followed by 40 cycles at $95^{\circ} \mathrm{C}$ for $10 \mathrm{sec}, 58^{\circ} \mathrm{C}$ for $20 \mathrm{sec}$ and $72^{\circ} \mathrm{C}$ for $15 \mathrm{sec}$. The relative expression levels of miR-451 and SAMD4B were normalized to RNU6 and GAPDH, respectively. The $2^{-\Delta \Delta \mathrm{Cq}}$ method (28) was used to analyze the relative fold changes.

The Cancer Genome Atlas (TCGA) database. Using data from TCGA the top 100 patients with high expression and the bottom 100 patients with low expression were selected for analysis.

Apoptosis analysis. HCT116 cells were seeded in 6-well cell culture plates after $6 \mathrm{~h}$ of transfection. After $24 \mathrm{~h}$, the cells were harvested and then stained with Annexin V and PE according to the manufacturer's protocol (Biotium, Inc.). The cells were then analyzed using flow cytometry. Briefly, HCT-116 cells were seeded in 6 -well plates at a density of $5 \times 10^{5}$ cells $/ \mathrm{ml}$. After $12 \mathrm{~h}$ incubation at $37^{\circ} \mathrm{C}$, the HCT-116 cells in the experimental group were treated with miR-451 mimics, miR-451 inhibitor or miR-451 mimics + SAMD4B, and the cells were collected following $24 \mathrm{~h}$ incubation at $37^{\circ} \mathrm{C}$. A total of $5 \mu \mathrm{l}$ Annexin V-FITC and PE were added. Samples were left to stand in the dark at room temperature for $15 \mathrm{~min}$ and then analyzed for apoptosis by flow cytometry. The experiments were repeated three times. The total percentage of apoptotic cells is presented as the sum of the apoptotic cell populations in the early and late stages. CytoFLEX flow cytometer was used for detection and CytExpert 2.4 software (both Beckman Coulter, Inc.) was used for data analysis.

Transwell assays. Cells were stained with a $0.1 \%$ crystal violet solution. The assay uses $8.0 \mu \mathrm{m}$ Transwell inserts (Corning, Inc.). The Transwell assay protocol was performed as described by Zhu et al (29). In brief, cells were digested and collected after $24 \mathrm{~h}$ transfection, then resuspended in DMEM (Sigma-Aldrich; Merck KGaA) containing 1\% FBS. The cell density was adjusted density to $1 \times 10^{5} / \mathrm{ml}$; then $200 \mu \mathrm{l}$ cell suspension were added to the upper chamber, and $600 \mu \mathrm{l}$ $10 \%$ FBS was added to the lower chamber before incubation at $37^{\circ} \mathrm{C}$. After $24 \mathrm{~h}$, cells on the upper layer of the Transwell cell filter membrane were removed using absorbent cotton; the filter membrane was fixed in 4\% paraformaldehyde for $30 \mathrm{~min}$ at room temperature and stained with $1 \%$ crystal violet for $15 \mathrm{~min}$ at room temperature. The slides were viewed and images captured under a light microscope (Olympus Corporation) at x100 magnification.

Luciferase reporter assay. A dual-luciferase assay system (Promega Corporation) was applied to measure luciferase activity. The primer sequences for amplifying the 3'-UTR of SAMD4B were: SAMD4B-wild-type (WT) forward (F): 5'-CTCACTGGCGGACTGCAAT-3; reverse(R): 5'-GTAGCC TCATGTACTCCGACTT-3'. Mutation primers were designed based on miR-451 and the SAMD4B gene 3 'UTR sequence binding mutation site. SAMD4B mutant (Mut) F: 5'-CTCTCC CTGCCACTGTCTTG-3'; R:5'-ATTCTGCAAGGACAGGAG CC-3'. The 3'-UTR segments of SAMD4B that were predicted to interact with miR-451 were amplified from human genomic DNA via PCR and inserted into the HindIII and SacI sites of the miR-451 expression reporter vector. The dual luciferase assay (Promega Corporation) was performed according to the manufacturer's protocol. The Luciferase reporter vector was pGL3-Basic (Youbio) and Renilla luciferase was used for normalization. Lipofectamine 3000 (Thermo Fisher Scientific, Inc.) was used to transfect the plasmid and mimics. After $72 \mathrm{~h}$ transfection, dual luciferase assay (Promega Corporation) was performed according to the manufacturer's protocol.

Western blot analysis. Cells were lysed in ice-cold RIPA buffer (cat. no. P0013B; Beyotime Institute of Biotechnology). The cells lysates were centrifuged at $12,000 \times \mathrm{g}$ for $15 \mathrm{~min}$ at $4^{\circ} \mathrm{C}$ and the supernatants were stored at $-80^{\circ} \mathrm{C}$. The protein concentration was determined via BCA method. The protein extracts were fractionated by electrophoresis on $10 \%$ sodium dodecyl sulfate-polyacrylamide gel, transferred onto PVDF membranes and blocked with 5\% non-fat milk powder for $2 \mathrm{~h}$ at room temperature. Then the membranes were incubated overnight at $4^{\circ} \mathrm{C}$ with primary antibodies as follows: Anti-SAMD4B (ProteinTech Group, Inc.; 1:800; cat. no. 17723-1-AP), anti-Bax (ProteinTech Group, Inc.; 1:2,000; cat. no. 50599-2-Ig), anti-Bcl2 (Abcam; 1:2,000; cat. no. ab182858) and anti-GAPDH (ProteinTech Group, Inc.; 1:10,000; cat. no. 10494-1-AP). After washing with $1 \mathrm{X}$ TBST 
Table II. Clinical features of patients and controls enrolled in the present study.

\begin{tabular}{lcccr}
\hline Characteristic & CRC patients $(\mathrm{n}=50)$ & Control $(\mathrm{n}=50)$ & $\chi^{2 / \mathrm{t}}$ & P-value \\
\hline Age (mean \pm standard deviation) & $62.0 \pm 10.4$ & $60.8 \pm 14.2$ & 0.457 & 0.648 \\
Sex & & & 0.167 & 0.683 \\
Male & 31 & 29 & & \\
Female & 19 & 21 & & \\
\hline
\end{tabular}

Table III. The primer and sequence information used for reverse transcription-quantitative PCR.

\begin{tabular}{ll}
\hline Gene & \multicolumn{1}{c}{ Sequence } \\
\hline SAMD4B & F: 5'-CTCACTGGCGGACTGCAAT-3' \\
GAPDH & R: 5'-GTAGCCTCATGTACTCCGACTT-3' \\
miR-451 & F: 5'-GTCGATGGCTAGTCGTAGCATCGAT-3' \\
U6 & R: 5'-TGCTAGCTGGCATGCCCGATCGATC-3' \\
miR-451 mimics & F: 5'-TCCGATTGAGTCATTACCAT-3' \\
& R: 5'-GTGCAGGGTCCGAGGT-3' \\
& F: 5'-TAAGATCGTGAAGCGTTC-3' \\
& R: 5'-GTGCAGGGTCCGAGGT-3' \\
& F: 5'-AAACCGUUACCAUUACUGAGUU-3' \\
& R: 3'-CUCAGUAAUGGUAACGGUUUUU-5'
\end{tabular}

F, forward; R, reverse; $\mathrm{miR}$, microRNA.

( $0.1 \%$ Tween) buffer four times, the blots were incubated with horseradish peroxidase-conjugated Goat Anti-Rabbit IgG(H+L; ProteinTech Group, Inc.; 1:2,000; cat. no. SA00001-2) at $4^{\circ} \mathrm{C}$ for $1 \mathrm{~h}$. Exposure imaging was performed using the Bio-Rad chemiluminescence imaging system (Bio-Rad Laboratories, Inc.). The blot optical density was determined by Image Lab software 5.2.1 (Bio-Rad Laboratories, Inc.).

Immunohistochemistry and Immunofluorescence Staining. The IHC assays were performed as described by Yamadera et al (30). In brief, the implanted xenograft tumor tissues were excised and fixed with $4 \%$ paraformaldehyde for $24 \mathrm{~h}$ at room temperature, dehydrated using a graded alcohol series of $60,70,80,90,95$ and $100 \%$ ethanol and finally embedded in paraffin. The $5-\mu \mathrm{m}$-thick paraffin sections were dewaxed and dehydrated, incubated with sealed serum and human rabbit ki-67 primary antibodies (1:400; cat. no. 9027; CST), were added in a wet box at $4^{\circ} \mathrm{C}$ overnight. After washing with PBS, sections were incubated with secondary antibodies (ProteinTech Group, Inc.; 1:1,000; cat. no. SA00004-2) at room temperature for $1 \mathrm{~h}$ and positive expression was detected with a DAB kit (Beyotime Institute of Biotechnology) at room temperature for $30 \mathrm{~min}$. The nuclei were stained with hematoxylin at room temperature for $8 \mathrm{~min}$. The slides were viewed and images captured under a light microscope (Olympus Corporation) at 200x magnification.

When conducting immunofluorescence experiments, HCT116 cells were seeded in 24-well cell culture plates after $6 \mathrm{~h}$ of transfection. The culture medium was removed after $24 \mathrm{~h}$, cells were fixed with $4 \%$ paraformaldehyde at room temperature for $20 \mathrm{~min}$ and permeabilized with $0.5 \%$ Triton $\mathrm{X}-100$ at room temperature for $20 \mathrm{~min}$ and then blocked with $5 \%$ blocking solution at room temperature for $2 \mathrm{~h}$. Following a phosphate-buffered saline (PBS) wash, the cells were then incubated with an anti-SAMD4B (ProteinTech Group, Inc.; 1:50; cat. no. 17723-1-AP) antibody overnight at $4^{\circ} \mathrm{C}$. The cells were then incubated in the dark with a goat anti-rabbit IgG-FITC antibody (ProteinTech Group, Inc.; 1:50; cat. no. SA00003-2) at room temperature for $1 \mathrm{~h}$ and cell nuclei were counterstained with DAPI at room temperature for $5 \mathrm{~min}$. Cells were reviewed and images captured with a fluorescence microscope (Olympus Corporation) at x400 magnification

Statistical analysis. The data were expressed as the means \pm SD and analyzed using SPSS 23 statistical software (IBM Corp.). Unpaired Student's t-tests or one-way ANOVA with Dunnett's or Bonferroni post hoc tests were performed to determine statistical significance. Categorical variables were analyzed using $\chi^{2}$ test or Fisher's exact test. The Kaplan-Meier method was applied to assess the survival rate of patients with $\mathrm{CRC}$. $\mathrm{P}<0.05$ was considered to indicate a statistically significant difference.

\section{Results}

Differential expression of miR-451 in patients with $C R C$ and cell lines. miR-451 expression was significantly decreased in patients with CRC when compared with serum from normal individuals (Fig. 1A). In addition, data from TCGA demonstrated that decreased miR-451 expression was notably 
A

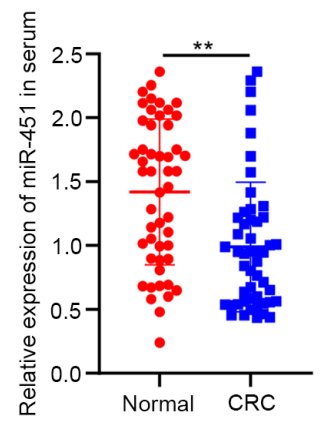

B

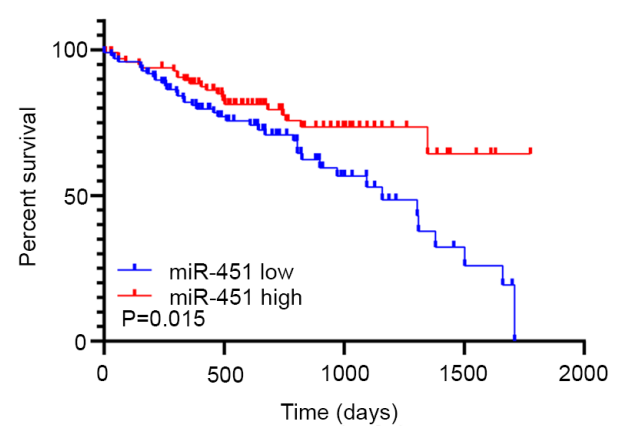

C

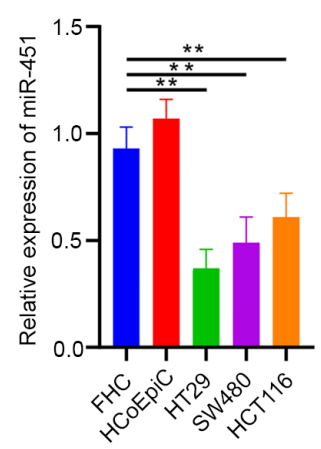

Figure 1. Relative miR-451 expression in patients with CRC and CRC cells. (A) RT-qPCR demonstrated the relative expression of miR-451 in patients with CRC $(n=50)$ and normal samples $(n=50) .{ }^{* *} \mathrm{P}<0.01$ vs. normal control group. (B) Kaplan-Meier analysis demonstrated the relationship between miR-451 expression and overall survival of patients with CRC based on The Cancer Genome Atlas data. (C) RT-qPCR demonstrated the relative expression of miR-451 in different types of CRC cell lines and normal colorectal mucosal cell lines. ${ }^{* *} \mathrm{P}<0.01$ vs. FHC cells. miR, microRNA; CRC, colorectal cancer; RT-qPCR, reverse transcription-quantitative PCR.

associated with shorter overall survival rate of patients with CRC (Fig. 1B). In addition, miR-451 expression was lower in CRC cell lines (HT29, SW480 and HCT116) compared with the normal colorectal mucosal cell lines (FHC and HCoEpiC) (Fig. 1C).

miR-451 suppresses CRC cell proliferation and migration, increases sensitivity to chemotherapy and promotes apoptosis. To explore the role of miR-451 in the tumorigenesis of CRC, the HCT116 cell line, which exhibits intermediate expression of miR-451, was selected for further study. Mimics and inhibitors specific to miR-451 were transfected into HCT116 cells and miR-451 expression detected $72 \mathrm{~h}$ post transfection (Fig. 2A). Cell viability was notably decreased in the miR-451mimic groups but increased in the miR-451-inhibited cells (Fig. 2B). Overexpression of miR-451 increased oxaliplatin and 5-FU-induced inhibition of CRC cell growth and increased sensitivity to chemotherapy (Fig. 2C and D). When miR-451 was overexpressed, the number of apoptotic cells increased significantly, whereas less apoptosis was noted in the inhibitor group (Fig. 2E). Western blotting results demonstrated that expression of the apoptosis-inhibitor protein $\mathrm{Bcl}-2$ was downregulated but the apoptosis-promoting protein Bax was upregulated, supporting the conclusion that miR-451 promoted apoptosis (Fig. 2F). Additionally, results from the Transwell assay also demonstrated a lower migration rate in cells where miR-451 was overexpressed, but higher migration rates were observed when miR-451 was knocked down (Fig. 2G).

SAMD4B is a target of $m i R-451$. To investigate how miR-451 regulates the malignant biological behavior of CRC cells, target gene candidates of miR-451 were searched for (TargetScan, targetscan.org/; microRNA, microRNA.org/ and mirdb, mirdb.org/) and SAMD4B was identified as a potential candidate (Fig. 3A and B). To explore the regulatory effect of miR-451 on SAMD4B, HCT116 cells were transfected with the miR-451 mimics or inhibitors. After $72 \mathrm{~h}$ transfection, SAMD4B expression was measured by RT-qPCR and western blotting. Results demonstrated that overexpression of miR-451 significantly downregulated the expression of SAMD4B at the mRNA and protein levels and inhibition of miR-451 expression had the opposite effect (Fig. 3C and D). These results were confirmed using immunofluorescence (Fig. 3E). Furthermore, miR-451 directly targeted the 3'-UTR of SAMD4B as determined by luciferase reporter assay (Fig. 3F). These findings indicated that miR-451 inhibited SAMD4B mRNA and protein expression by directly targeting its 3'-UTR.

miR-451 regulates growth and migration via SAMD4B in $C R C$. Migration and malignant proliferation are important factors in the progression and recurrence of CRC. Plasmids specific to SAMD4B were transfected into HCT116 cells and SAMD4B mRNA and protein levels analyzed $72 \mathrm{~h}$ post transfection (Fig. 4A). The present study then explored whether miR-451 was closely linked to the regulation of CRC proliferation and migration via SAMD4B. The cell viability analysis results demonstrated that overexpression of SAMD4B restored the cell proliferation inhibited by miR-451 (Fig. 4B) and overexpression of SAMD4B decreased the oxaliplatin and 5-FU chemosensitivity induced by miR-451 (Fig. 4C and D). Flow cytometric analysis revealed that the overexpression of SAMD4B attenuated miR-451-induced apoptosis (Fig. 4E). Consistent with these results, analysis of apoptotic protein expression via western blotting further confirmed the above results (Fig. 4F). In addition, Transwell experiments suggested that the overexpression of SAMD4B inhibited miR-451-induced effects on cell migration (Fig. 4G). Taken together, these results illustrate that miR-451 regulated proliferation and migration through SAMD4B in CRC cells.

miR-451 inhibits CRC growth through SAMD4B in vivo. In the animal experiment, it was observed that miR-451 overexpression resulted in a significant decrease in tumor volume (Fig. 5A) and tumor weight (Fig. 5B). When both miR-451 and SAMD4B were overexpressed, SAMD4B overexpression reversed inhibition of tumor growth induced by miR-451. The IHC experiment demonstrated that the $\mathrm{Ki}-67$ protein was dramatically decreased in the miR-451 group but overexpressed in the SAMD4B group and co-transfection of miR-451 and SAMD4B reversed inhibition of Ki-67 expression compared with miR-451 expression alone (Fig. 5C). The TCGA database was used to identify the expression of SAMD4B in CRC 


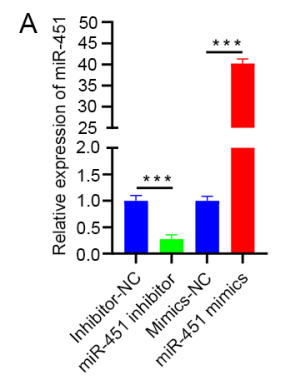

B

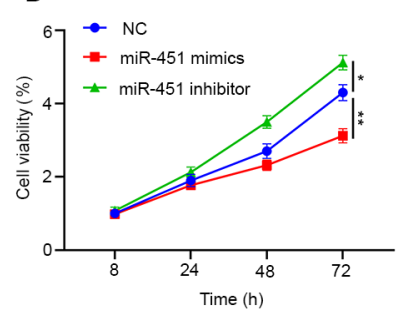

$\mathrm{C}$

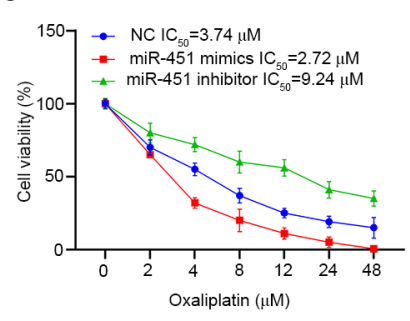

D

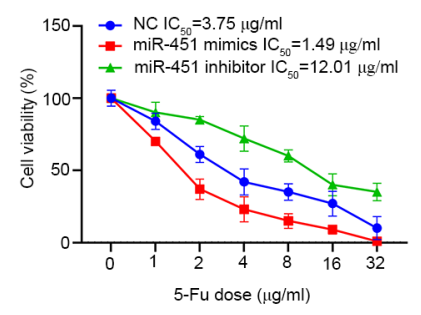

E

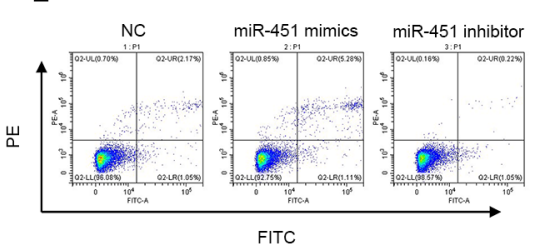

G

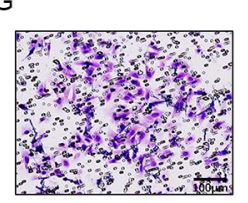

NC
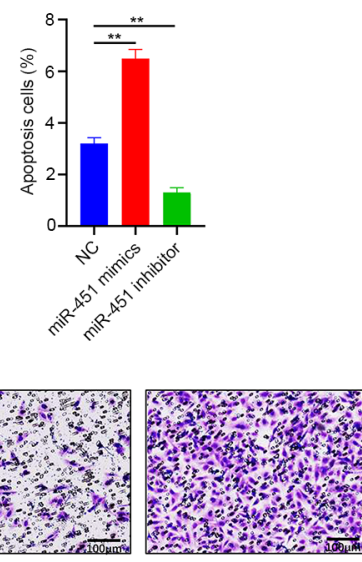

miR-451 inhibitor

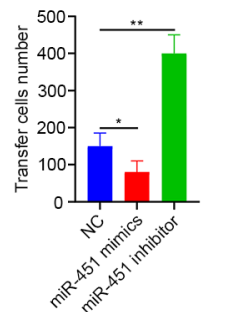

F

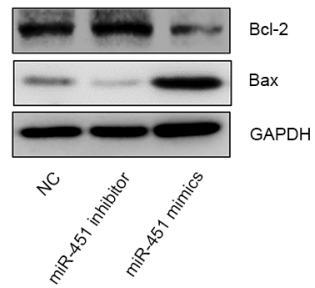

Figure 2. miR-451 suppressed CRC cell proliferation and migration, increased sensitivity to chemotherapy and promoted apoptosis. (A) RT-qPCR detected miR-451 expression at $72 \mathrm{~h}$ post transfection of miR-451 mimics or inhibitor in HCT116 cells. (B) CCK-8 assays demonstrated decreased proliferation in HCT116 cells in the miR-451 mimics group compared with the NC and miR-451 inhibitor groups. Overexpression of miR-451 increased (C) oxaliplatin and (D) 5-FU inhibition of CRC cell growth. (E) Flow cytometric apoptosis analysis demonstrated that more cell apoptosis occurred in the miR-451 mimics group. Histograms show the percent of apoptotic cells in different groups. (F) Western blotting results show that miR-451 affected the protein expression of Bcl-2 and Bax. (G) Transwell assay demonstrated the migration of HCT116 cells. Histograms show the number of migrated cells in different groups (magnification, $\mathrm{x} 100$ ). ${ }^{*} \mathrm{P}<0.05,{ }^{* *} \mathrm{P}<0.01$ and ${ }^{* * * *} \mathrm{P}<0.001$ vs. NC group. miR, microRNA; CRC, colorectal cancer; RT-qPCR, reverse transcription-quantitative PCR; NC, normal control.

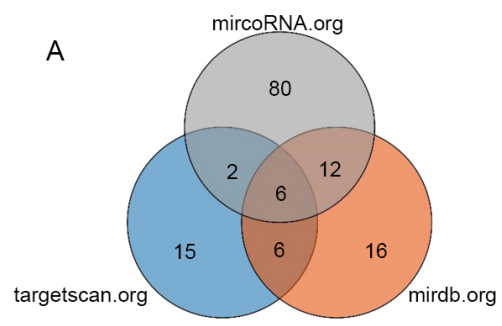

B

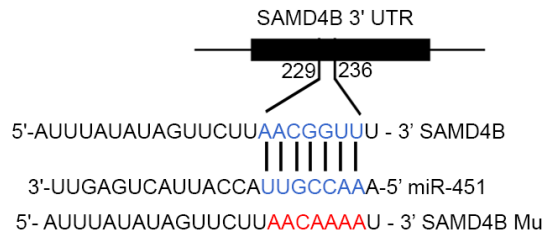

C

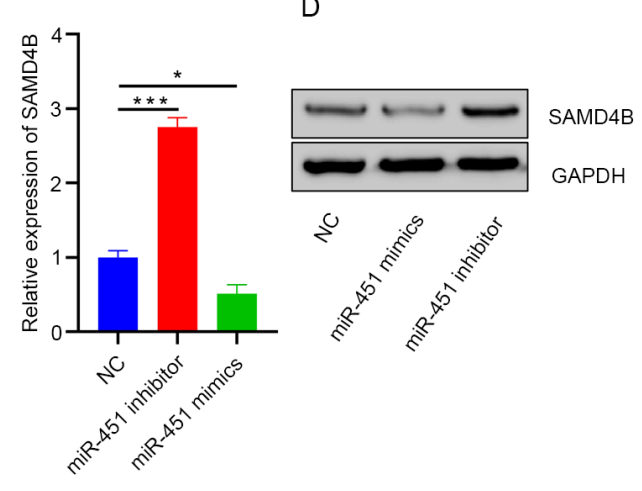

$\mathrm{E}$

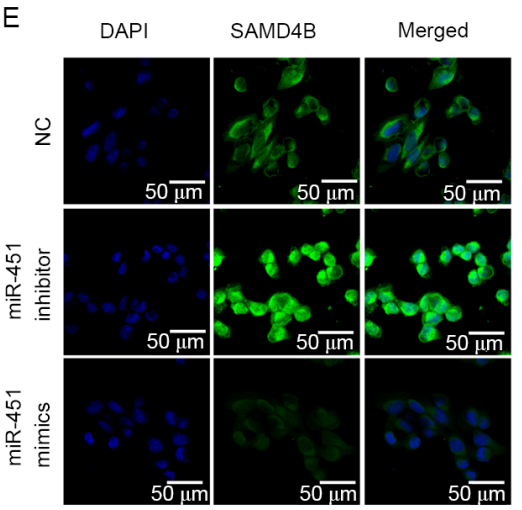

$\mathrm{F} \quad \mathrm{NC}$

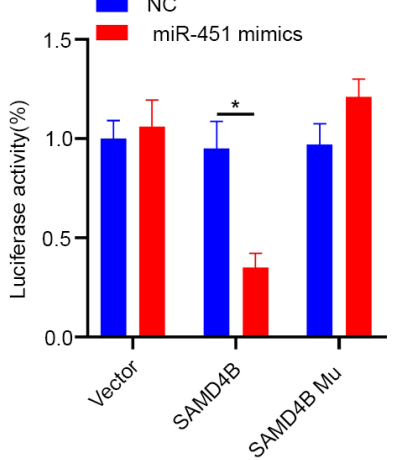

Figure 3. SAMD4B is a direct target gene of miR-451 in CRC cells. (A) Search for target gene candidates of miR-451 using mirdb.org, microRNA.org and targetscan.org. (B) Schematics of miR-451, SAMD4B WT and Mut luciferase reporter plasmids. (C) RT-qPCR analysis demonstrated the relative expression of SAMD4B (vs. GAPDH) in HCT116 cells transfected with miR-451 mimics or miR-451 inhibitor. (D) Western blotting results demonstrated that miR-451 affected the SAMD4B protein expression. (E) Immunofluorescence assay demonstrated that SAMD4B expression was downregulated by the overexpression of miR-451. (F) Analysis of luciferase activation in treated HCT116 cells. " $\mathrm{P}<0.05$ and ${ }^{* * * *} \mathrm{P}<0.001$ vs. the NC group. miR, microRNA; CRC, colorectal cancer; WT, wild-type; Mut, mutant; RT-qPCR, reverse transcription-quantitative PCR; NC, normal control. 
A

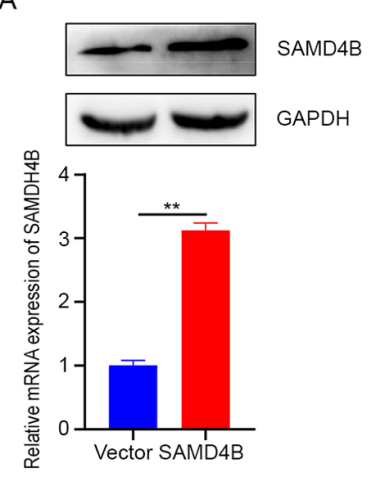

E

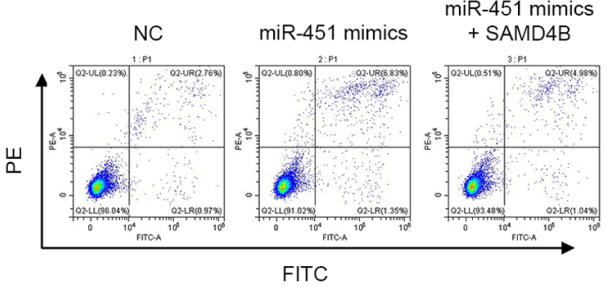

G

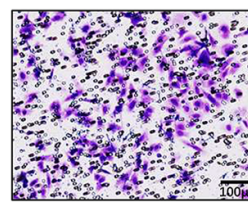

NC
B

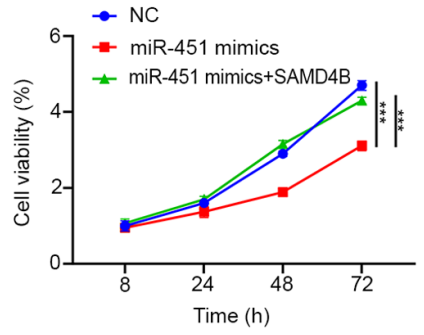

C

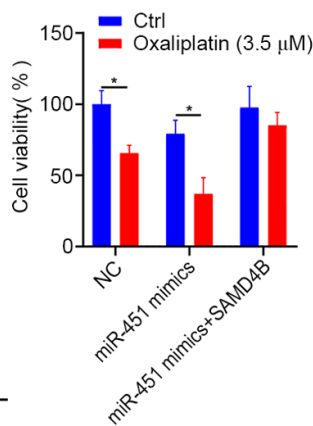

D

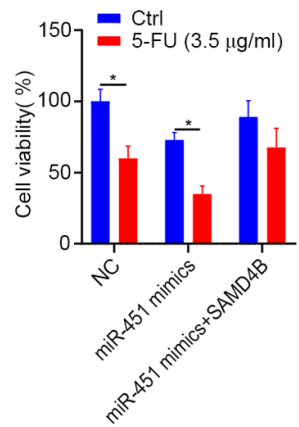

F
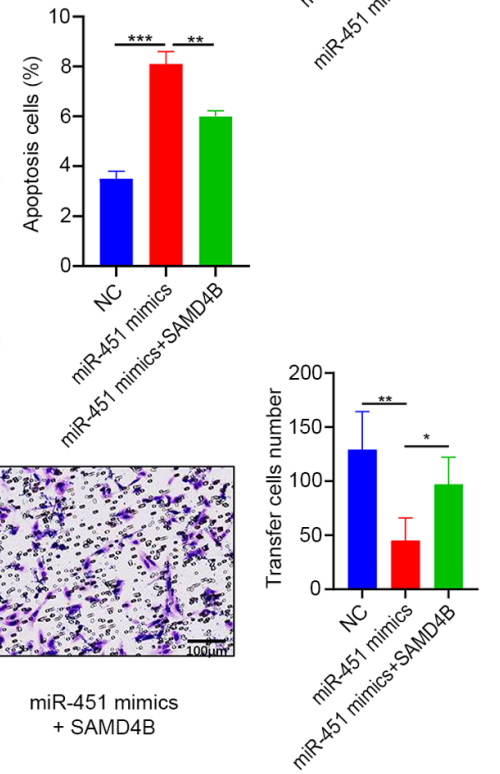

Figure 4. miR-451 inhibited the malignant biological properties of CRC cells by suppressing SAMD4B. (A) Western blotting and reverse transcriptionquantitative PCR detected SAMD4B expression at $72 \mathrm{~h}$ post transfection of the SAMD4B plasmid in HCT116 cells. (B) CCK-8 assays demonstrating overexpression of SAMD4B restored cell proliferation inhibited by miR-451. Overexpression of SAMD4B decreased the (C) oxaliplatin and (D) 5-FU chemosensitivity induced by miR-451. (E) Flow cytometric apoptosis analysis demonstrating overexpression of SAMD4B attenuated miR-451-induced cell apoptosis. Histograms show the percentage of apoptotic cells in different groups. (F) Western blotting analysis of Bcl-2 and Bax protein levels in HCT116 cells transfected with miR-451 mimics or miR-451 mimics+SAMD4B. (G) Transwell assay demonstrating the relative number of migrating cells in NC, miR-451 mimics and miR-451+SAMD4B groups (magnification, $\mathrm{x} 100$ ). ${ }^{*} \mathrm{P}<0.05,{ }^{* * *} \mathrm{P}<0.01$ and ${ }^{* * * *} \mathrm{P}<0.001$ vs. the NC group. miR, microRNA; CRC, colorectal cancer; $\mathrm{NC}$, normal control; Ctrl, control.

and paracancer tissues and found that SAMD4B is highly expressed in both types of tumor tissues (Fig. 5D). SAMD4B is significantly related to poor prognosis in patients with CRC (Fig. 5E). In summary, these findings supported the hypothesis that miR-451 is essential to inhibition of malignant behavior via targeting SAMD4B (Fig. 5F).

\section{Discussion}

$\mathrm{CRC}$ is a common malignant tumor of the digestive system. The pathological development of CRC is a long, multi-step process progressing from benign precancerous lesions (adenoma) to malignant tumors (adenocarcinoma) (31). The treatment of CRC involves surgical resection of the primary lesion combined with chemotherapy, radiotherapy and/or molecular targeted therapy (32). Chemotherapeutic drugs based on 5-FU and oxaliplatin do not sufficiently control the progression of advanced CRC (33). Molecular targeted drugs such as EGFR/VEGFR inhibitors offer hope for patients with advanced CRC (34), but the prognosis of advanced patients with CRC remains poor. Conventional tumor markers such as CEA and CA19-9 cannot suitably predict the occurrence and development of tumors due to poor sensitivity (1). In recent years, researchers have been searching for valuable markers for the diagnosis, treatment and prediction of CRC (35).

In 2005, Altuvia et al (36) searched miRNA precursor sequences near known miRNA genes. He used bioinformatics to first identify an miRNA adjacent to miR-144 and named it miR-451 (36). Subsequently, a growing number of studies focused on miR-451 in living organisms have been published. Regarding physiological processes, miR-451 is involved in hematopoietic differentiation (37-39), embryo maturation (40) and human nervous system development (41). In pathological processes, miR-451 has been shown to improve cardiac hypertrophy (42) and attenuate ischemic brain infarction (43).

In oncology, miR-451 has significance in different types of tumors such as lung cancer, pancreatic cancer and glioma $(19,20,44)$. In CRC, miR-451 serves an important role. Mamoori et al (24) found that elevated expression of miR-451 contributed to increased apoptosis, reduced proliferation and changed cell cycle distribution. Vidal et al (45) evaluated miRNA profiles in embryonic samples and cancer cell lines. They concluded that miR-451 is highly expressed in placental samples but is significantly lower in cancer 

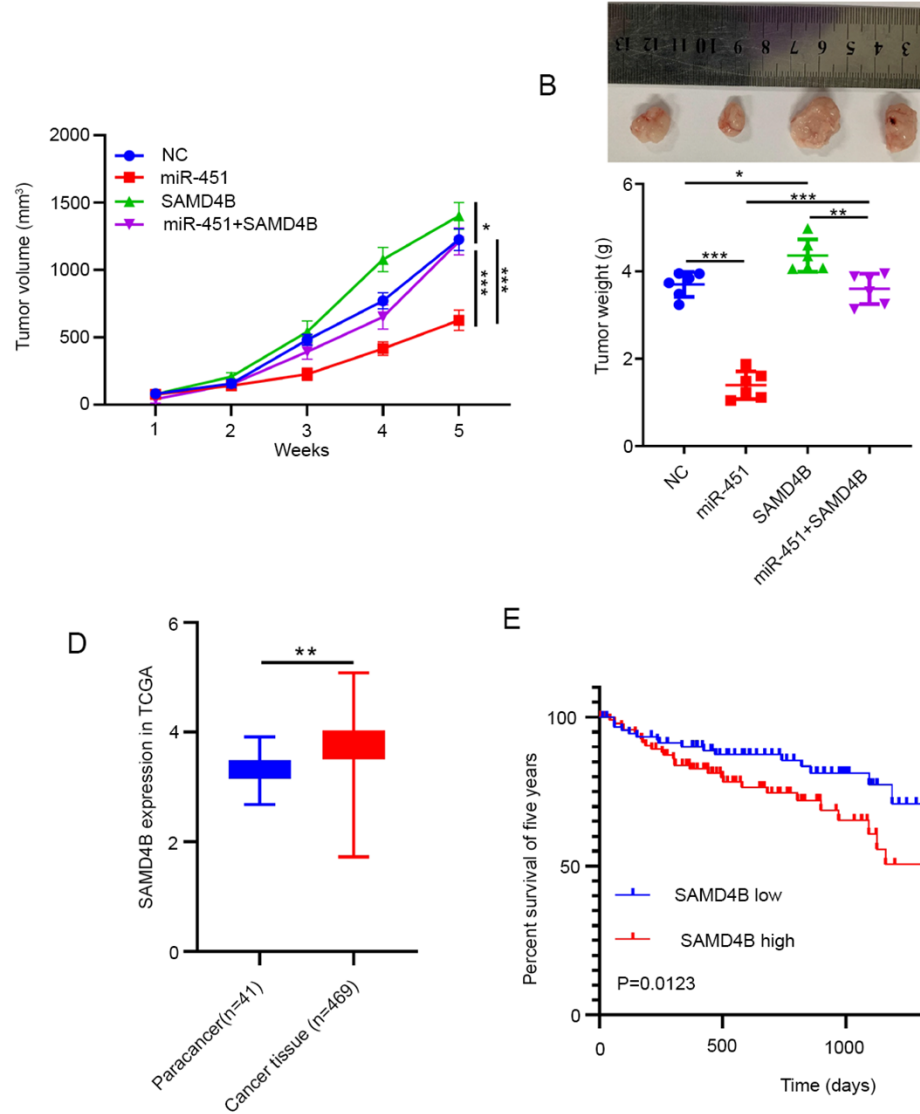

E

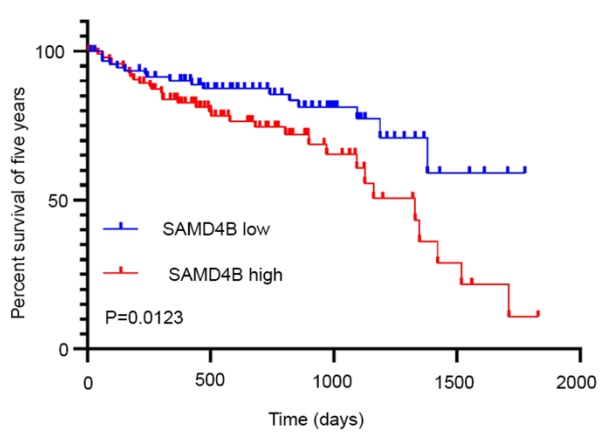

C

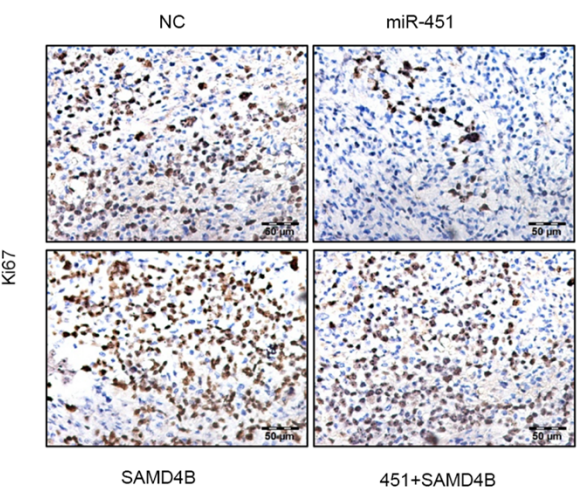

$\mathrm{F}$

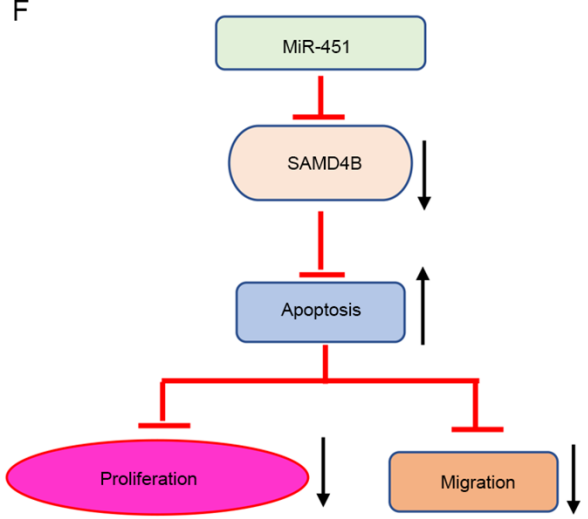

Figure 5. miR-451 inhibited CRC growth via SAMD4B in vivo. (A) Growth curve of subcutaneously implanted tumors. (B) Surgically excised xenograft tumor tissues at 5 weeks after initial implantation. (C) The IHC assay detected the expression of Ki-67 in different groups of animal samples. (D) Expression of SAMD4B in CRC and paracancerous tissues and (E) its effect on survival of patients with CRC, based on the data of The Cancer Genome Atlas. (F) miR-451 is essential to blocking CRC malignant behavior via targeting SAMD4B. ${ }^{*} \mathrm{P}<0.05,{ }^{* * *} \mathrm{P}<0.01$ and ${ }^{* * * *} \mathrm{P}<0.001$ vs. NC group. miR, microRNA; CRC, colorectal cancer; $\mathrm{NC}$, normal control.

cell lines. Transfection of miR-451 significantly improves the malignant biological behavior of the HT-29 CRC cell line by decreasing proliferation, migration, invasion and colony formation (45). Consistent with these studies, the present study also demonstrated overexpression of miR-451 sensitized CRC cells to oxaliplatin and 5-FU. Expression of the apoptosis-inhibitory protein Bcl-2 was downregulated whereas the apoptosis-promoting protein Bax was upregulated, supporting the hypothesis that miR-451 promoted apoptosis. miR-451 also reduced cell migration. In animal experiments, miR-451 reduced the growth of xenograft tumors, decreased tumor volume and tumor weight and IHC staining indicated a decrease in Ki-67 expression. These results suggest that the proliferative capacity of the tumors was suppressed.

The present study further explored the internal tumor suppressor mechanisms of miR-451 in CRC. In vitro and in vivo functional experiments confirmed that miR-451 suppressed the malignant biological behavior of CRC cells by targeting SAMD4B.

In conclusion, the present study identified miR-451 as a predictive miRNA that is closely associated with longer survival rates in patients with CRC. Through gain and loss-of-function studies, the present study concluded that miR-451 inhibited proliferation and migration, enhanced the sensitivity of CRC cells to chemotherapy and probably mediated its effects by targeting SAMD4B. The present study illustrated the potential value of miR-451 and its target gene SAMD4B in predicting the progression and prognosis of patients with CRC. The miR-451/SAMD4B axis may serve as a novel therapeutic target in patients with CRC.

\section{Acknowledgements}

Not applicable.

\section{Funding}

The present study was supported by Natural Science Foundation of Chongqing, China (grant no. cstc2020jcyj-msxmX0183), District Science and Technology Commission Project of Chongqing Jiangjin (grant nos. Y2019067 and Y2020050) and Health and Family Planning Commission Medical Research Project of Chongqing (grant no. 2017MSXM171).

\section{Availability of data and materials}

The data of this study are available from the corresponding author on reasonable request. 


\section{Authors' contributions}

CW and XL contributed equally to the present study. CW and XL designed the study and drafted the manuscript; BL interpreted the data and performed statistical analysis; GS and CP participated in the coordination of the study; and DX supervised the study and helped to draft the manuscript. All authors contributed toward data analysis and critically revising the paper and agree to be accountable for all aspects of the work. CW and XL confirmed the authenticity of all the raw data. All authors read and approved the final manuscript.

\section{Ethics approval and consent to participate}

The Ethics Committee of the Jiangjin Central Hospital of Chongqing approved the research protocol (approval no. 20190611-28) and informed and written consent was received from each patient. Animal procedures were approved by the Ethics Committee of Chongqing Medical University (approval no. 2019-256).

\section{Patient consent for publication}

Not applicable.

\section{Competing interests}

The authors declare that they have no competing interests.

\section{References}

1. Das V, Kalita $\mathbf{J}$ and Pal M: Predictive and prognostic biomarkers in colorectal cancer: A systematic review of recent advances and challenges. Biomed Pharmacother 87: 8-19, 2017.

2. Wang FW, Cao CH, Han K, Zhao YX, Cai MY, Xiang ZC, Zhang JX, Chen JW, Zhong LP, Huang Y, et al: APC-activated long noncoding RNA inhibits colorectal carcinoma pathogenesis through reduction of exosome production. J Clin Invest 129 727-743, 2019.

3. Dy GK, Hobday TJ, Nelson G, Windschitl HE, O'Connell MJ, Alberts SR, Goldberg RM, Nikcevich DA and Sargent DJ: Long-term survivors of metastatic colorectal cancer treated with systemic chemotherapy alone: A North Central Cancer Treatment Group review of 3811 patients, N0144. Clin Colorectal Cancer 8: 88-93, 2009.

4. Skarkova V, Kralova V, Vitovcova B and Rudolf E: Selected aspects of chemoresistance mechanisms in colorectal carcinoma-a focus on epithelial-to-mesenchymal transition, autophagy, and apoptosis. Cells 8: 234, 2019.

5. Chen CZ, Li L, Lodish HF and Bartel DP: MicroRNAs modulate hematopoietic lineage differentiation. Science 303: 83-96, 2004

6. Lee SD, Yu D, Lee DY, Shin HS, Jo JH and Lee YC: Upregulated microRNA-193a-3p is responsible for cisplatin resistance in CD44(+) gastric cancer cells. Cancer Sci 110: 662-673, 2019.

7. Ding HX, Lv Z, Yuan Y and Xu Q: miRNA polymorphisms and cancer prognosis: A systematic review and meta-analysis. Front Oncol 8: 596, 2018.

8. Rodríguez-Martínez A, de Miguel-Pérez D, Ortega FG, García-Puche JL, Robles-Fernández I, Exposito J, Martorell-Marugan J, Carmona-Sáez P, Garrido-Navas MDC, Rolfo C, et al: Exosomal miRNA profile as complementary tool in the diagnostic and prediction of treatment response in localized breast cancer under neoadjuvant chemotherapy. Breast Cancer Res 21: 21, 2019.

9. Vychytilova-Faltejskova P and Slaby O: MicroRNA-215: From biology to theranostic applications. Mol Aspects Med 70: 72-89, 2019.
10. Wang R, Sun Y, Yu W, Yan Y, Qiao M, Jiang R, Guan W and Wang L: Downregulation of miRNA-214 in cancer-associated fibroblasts contributes to migration and invasion of gastric cancer cells through targeting FGF9 and inducing EMT. J Exp Clin Cancer Res 38: 20, 2019.

11. Hou GL, Xu WF, Jin YB, Wu J, Pan Y and Zhou F: miRNA-217 accelerates the proliferation and migration of bladder cancer via inhibiting KMT2D. Biochem Biophys Res Commun 519: 747-753, 2019.

12. Liu AN, Qu HJ, Gong WJ, Xiang JY, Yang MM and Zhang W: LncRNA AWPPH and miRNA-21 regulates cancer cell proliferation and chemosensitivity in triple-negative breast cancer by interacting with each other. J Cell Biochem 120: 14860-14866, 2019.

13. Shivapurkar N, Weiner LM, Marshall JL, Madhavan S, Deslattes Mays A, Juhl H and Wellstein A: Recurrence of early stage colon cancer predicted by expression pattern of circulating microRNAs. PLoS One 9: e84686, 2014.

14. Allen B, Schneider A, Victoria B, Nunez Lopez YO, Muller M, Szewczyk M, Pazdrowski J, Majchrzak E, Barczak W, Golusinski W, et al: Blood serum from head and neck squamous cell carcinoma patients induces altered microRNA and target gene expression profile in treated cells. Front Oncol 8: 217, 2018.

15. Zhao L, Yang S, You X, He W and Xue J: Novel miRNA-based biomarker panel for detection $\beta 2$-agonists in goats. Food Chem 288: 15-21, 2019.

16. Usuba W, Urabe F, Yamamoto Y, Matsuzaki J, Sasaki H, Ichikawa M, Takizawa S, Aoki Y, Niida S, Kato K, et al: Circulating miRNA panels for specific and early detection in bladder cancer. Cancer Sci 110: 408-419, 2019.

17. Zheng D, Ding Y, Ma Q, Zhao L, Guo X, Shen Y, He Y, Wei W and Liu F: Identification of serum MicroRNAs as novel biomarkers in esophageal squamous cell carcinoma using feature selection algorithms. Front Oncol 8: 674, 2018.

18. Shen Y, Gong JM, Zhou LL and Sheng JH: Correction: miR-451 as a new tumor marker for gastric cancer. Oncotarget 10: 6396, 2019.

19. Liu Y, Li H, Li LH, Tang JB and Sheng YL: Mir-451 inhibits proliferation and migration of non-small cell lung cancer cells via targeting LKB1/AMPK. Eur Rev Med Pharmacol Sci 23 (Suppl 3): S274-S280, 2019.

20. Guo R, Gu J, Zhang Z, Wang Y and Gu C: miR-451 promotes cell proliferation and metastasis in pancreatic cancer through targeting CAB39. Biomed Res Int 2017: 2381482, 2017.

21. Kong W, Feng L, Yang M, Chen Q, Wang H, Wang X and Hou J: Prognostic value of microRNA-451 in various cancers: A meta-analysis. Pathol Res Pract 215: 152726, 2019.

22. Khordadmehr M,Jigari-Asl F, Ezzati H, Shahbazi R, Sadreddini S, Safaei S and Baradaran B: A comprehensive review on miR-451: A promising cancer biomarker with therapeutic potential. J Cell Physiol 234: 21716-21731, 2019.

23. Bai $\mathrm{H}$ and Wu S: miR-451: A novel biomarker and potential therapeutic target for cancer. Onco Targets Ther 12: 11069-11082, 2019.

24. Mamoori A, Wahab R, Vider J, Gopalan V and Lam AK: The tumour suppressor effects and regulation of cancer stem cells by macrophage migration inhibitory factor targeted miR-451 in colon cancer. Gene 697: 165-174, 2019.

25. Mamoori A, Gopalan V, Lu CT, Chua TC, Morris DL, Smith RA and Lam AK: Expression pattern of miR-451 and its target MIF (macrophage migration inhibitory factor) in colorectal cancer. J Clin Pathol 70: 308-312, 2017.

26. Fan $X$ and Zhao Y: miR-451a inhibits cancer growth, epithelial-mesenchymal transition and induces apoptosis in papillary thyroid cancer by targeting PSMB8. J Cell Mol Med 23: 8067-8075, 2019.

27. Zhu D, Fang C, He W, Wu C, Li X and Wu J: MicroRNA-181a inhibits activated B-cell-like diffuse large B-cell lymphoma progression by repressing CARD11. J Oncol 2019: 9832956, 2019.

28. Livak KJ and Schmittgen TD: Analysis of relative gene expression data using real-time quantitative PCR and the 2(-Delta Delta C(T)) method. Methods 25: 402-408, 2001.

29. Zhu G, Cheng Z, Huang Y, Zheng W, Yang S, Lin C and Ye J: MyD88 mediates colorectal cancer cell proliferation, migration and invasion via NF- $\mathrm{BB} / \mathrm{AP}-1$ signaling pathway. Int J Mol Med 45: 131-140, 2020.

30. Yamadera M, Shinto E, Kajiwara Y, Mochizuki S, Okamoto K, Shimazaki H, Hase K and Ueno H: Differential clinical impacts of tumour budding evaluated by the use of immunohistochemical and haematoxylin and eosin staining in stage II colorectal cancer. Histopathology 74: 1005-1013, 2019. 
31. Fearon ER and Vogelstein B: A genetic model for colorectal tumorigenesis. Cell 61: 759-767, 1990.

32. Mármol I, Sánchez-de-Diego C, Pradilla Dieste A, Cerrada E and Rodriguez Yoldi MJ: Colorectal carcinoma: A general overview and future perspectives in colorectal cancer. Int J Mol Sci 18: 197, 2017.

33. To KK, Tong CW, Wu M and Cho WC: MicroRNAs in the prognosis and therapy of colorectal cancer: From bench to bedside. World J Gastroenterol 24: 2949-2973, 2018.

34. Yalcin S, Trad D, Kader YA, Halawani H, Demir OG, Mall R, Meshcheryakov A, Nasr F, Nosworthy A, Osinsky D, et al: Personalized treatment is better than one treatment fits all in the management of patients with mCRC: A consensus statement. Future Oncol 10: 2643-2657, 2014.

35. Zhang X, Sun XF, Cao Y, Ye B, Peng Q, Liu X, Shen B and Zhang H: CBD: A biomarker database for colorectal cancer. Database (Oxford) 2018: bay046, 2018.

36. Altuvia Y, Landgraf P, Lithwick G, Elefant N, Pfeffer S, Aravin A, Brownstein MJ, Tuschl T and Margalit H: Clustering and conservation patterns of human microRNAs. Nucleic Acids Res 33: 2697-2706, 2005.

37. Xu P, Palmer LE, Lechauve C, Zhao G, Yao Y, Luan J, Vourekas A, Tan H, Peng J, Schuetz JD, et al: Regulation of gene expression by miR-144/451 during mouse erythropoiesis. Blood 133: 2518-2528, 2019.

38. Shokri G, Kouhkan F, Nojehdehi S, Soleimani M, Pourfathollah AA, Nikougoftar Zarif M, Tamaddon M and Obeidi N: Simultaneous regulation of miR-451 and miR-191 led to erythroid fate decision of mouse embryonic stem cell. Iran J Basic Med Sci 224: 432-438, 2019

39. Yao H, Ma Y and Huang LJ: Deletion of miR-451 curbs JAK2(V617F)-induced erythrocytosis in polycythemia vera by oxidative stress-mediated erythroblast apoptosis and hemolysis. Haematologica 105: e153-e156, 2020.
40. Li X, Zhang W, Fu J, Xu Y, Gu R, Qu R, Li L, Sun Y and Sun X: MicroRNA-451 is downregulated in the follicular fluid of women with endometriosis and influences mouse and human embryonic potential. Reprod Biol Endocrinol 17: 96, 2019.

41. Zhang Z, Chang H, Li Y, Zhang T, Zou J, Zheng X and Wu J: MicroRNAs: Potential regulators involved in human anencephaly. Int J Biochem Cell Biol 42: 367-374, 2010.

42. Gan M,Zheng T, Shen L, Tan Y,Fan Y, Shuai S, Bai L,Li X, Wang J, Zhang S and Zhu L: Genistein reverses isoproterenol-induced cardiac hypertrophy by regulating miR-451/TIMP2. Biomed Pharmacother 112: 108618, 2019.

43. Fu C, Chen S, Cai N, Liu Z, Wang P and Zhao J: Potential neuroprotective effect of miR-451 against cerebral ischemia/ reperfusion injury in stroke patients and a mouse model. World Neurosurg 130: e54-e61, 2019.

44. Ogawa D, Ansari K, Nowicki MO, Salińska E, Bronisz A and Godlewski J: MicroRNA-451 inhibits migration of glioblastoma while making it more susceptible to conventional therapy. Noncoding RNA 5: 25, 2019.

45. Vidal DO, Ramão A, Pinheiro DG, Muys BR, Lorenzi JCC, de Pádua Alves C, Zanette DL, de Molfetta GA, Duarte G and Silva WA Jr: Highly expressed placental miRNAs control key biological processes in human cancer cell lines. Oncotarget 9: 23554-23563, 2018.

This work is licensed under a Creative Commons Attribution-NonCommercial-NoDerivatives 4.0 International (CC BY-NC-ND 4.0) License. 